\title{
meso and racemo Additions in Propagation for Radical Polymerization of Dialkyl Fumarates I. Stereoregularity of Poly(dialkyl fumarate)s
}

\author{
Masahiro Yoshioka, Akikazu Matsumoto, and Takayuki Otsu* \\ Department of Applied Chemistry, Faculty of Engineering, Osaka City University, \\ Sugimoto, Sumiyoshi-ku, Osaka 558, Japan
}

(Received March 14, 1991)

\begin{abstract}
The polymerization temperature dependence of the stereochemical configuration of some poly(dialkyl fumarate)s as poly(substituted methylene) was investigated by the use of ${ }^{13} \mathrm{C}$ NMR spectroscopy in order to clarify the propagation process in radical polymerization of dialkyl fumarates. The probability of meso addition $\left(P_{m}\right)$ for polymerization of di-tert-butyl fumarate increased from 0.68 to 0.80 , when the polymerization temperature decreased from $120^{\circ} \mathrm{C}$ to $0^{\circ} \mathrm{C}$. On the other hand, it was revealed that $P_{m}$ for dimethyl fumarate and diisopropyl fumarate decreased by the decreasing polymerization temperature, and that it was almost independent of the temperature for methyl tert-butyl fumarate. From the differences in activation enthalpies and entropies for meso and racemo additions, the effect of the ester alkyl groups on the propagation manner was discussed.

KEY WORDS Dialkyl Fumarate / Radical Polymerization / Poly(substituted methylene) / ${ }^{13} \mathrm{C}$ Nuclear Magnetic Resonance / Stereoregularity / Activation Enthalpy / Activation Entropy /
\end{abstract}

It has been reported that dialkyl fumarates (DRF) with bulky ester alkyl groups homopolymerize readily to give high molecular weight polymers in a radical mechanism despite of the 1,2-disubstituted ethylene structure, and that the resulting poly(DRF)s consist of a semiflexible poly(substituted methylene) structure. $^{1-5}$ The polymerization reactivity (polymerization rate and molecular weight of the polymer) and the flexibility of poly(DRF)s depend intensively upon the structure (bulkiness) of the ester substituents, indicating that the polymerization of DRF shows unique characteristics different from ordinary vinyl polymerizations. It has been also revealed that stereochemical structure of poly(DRF)s is dependent of the structure of the ester alkyl groups. ${ }^{6,7}$

The tacticity of poly(DRF)s is dominated by both opening mode of carbon-to-carbon double bond and direction of monomer addition to the propagating polymer radical.
As each carbon atom in the main chain of poly(DRF) as poly(substituted methylene) is pseudo-asymmetric, the stereochemistry based on the methylene unit should be considered as is shown in Figure 1. When Bernoullian probability is applied to the stereochemistry of poly(DRF)s, two parameters, $P_{m}\left(P_{r}\right)$ and $P_{m}^{\prime}\left(P_{r}^{\prime}\right)$ are required, which represent the probability of meso (racemo) addition and opening, respectively. However, because the opening of double bond is restricted to trans mode for polymerization of DRF, i.e., $P_{m}^{\prime}=1$ and $P_{r}^{\prime}=0$, the stereoregularity of poly(DRF) can be determined with solely $P_{m} \cdot{ }^{6,7}$

In the previous paper, ${ }^{7}$ we investigated the $P_{m}$ values for polymerization of di-tert-butyl fumarate (DtBF), methyl tert-butyl fumarate (MtBF) and dimethyl fumarate (DMF) by the use of ${ }^{13} \mathrm{C}$ NMR spectroscopy, and confirmed that meso addition was preferable in the propagation of DRF having a tert-butyl group as is shown in Figure 2. Furthermore, pre- 
(A)
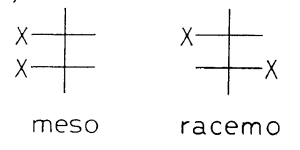

(B)
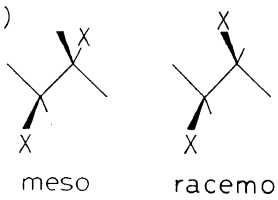

Figure 1. (A) Fischer and (B) planar zigzag projections of meso and racemo diads for poly(substituted methylene).

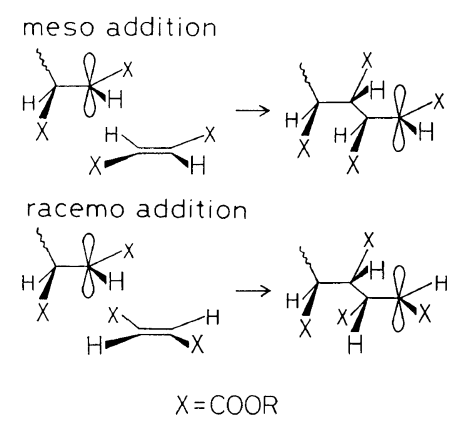

Figure 2. meso and racemo additions of DRF to a poly(DRF) radical, where the opening mode of double bond is restricted to trans (meso) mode (see the text).

liminary results indicated that meso addition was favored for polymerization of DtBF as the polymerization temperature decreased. Recently, we have found that temperature dependence of $P_{m}$ for other DRFs is different utterly from that for DtBF.

The purpose in this study is to examine the temperature dependence of $P_{m}$ for polymerization of various DRFs with the aid of ${ }^{13} \mathrm{C}$ NMR spectra of poly(DRF)s as a step for clarification of the addition manner of the monomer in propagation.

\section{EXPERIMENTAL}

DRFs were prepared and purified as reported previously..$^{-4} \mathrm{DtBF}$ : $\mathrm{mp} 71^{\circ} \mathrm{C}$, MtBF: bp $65^{\circ} \mathrm{C}(1 \mathrm{mmHg})$, DiPF: bp $93^{\circ} \mathrm{C}$ ( $7 \mathrm{mmHg}$ ), DMF: $\mathrm{mp} 102^{\circ} \mathrm{C} .2,2^{\prime}$-Azobisisobutyronitrile (AIBN), dimethyl 2,2'-azobisisobu- tyrate (MAIB), 1,1'-azobiscyclohexanecarbonitrile (ACN), di-tert-butyl peroxide (DTBPO) and tert-butyl hydroperoxide (TBHPO) were used as a radical initiator.

Polymerization was carried out in a degassed glass tube. The polymerization mixture was poured into a large amount of precipitant to isolate the polymer. Methanol, $n$-hexane, and diethyl ether were used as precipitant for poly(DtBF) or poly(DiPF), poly(MtBF), and poly(DMF), respectively. The polymer obtained was dried under vacuum. The purification of the polymers was performed by reprecipitation.

The number- and weight-average molecular weights $\left(\bar{M}_{n}\right.$ and $\bar{M}_{w}$, respectively $)$ and polydispersity $\left(\bar{M}_{w} / \bar{M}_{n}\right)$ of the resulting polymers were determined by gel permeation chromatography (GPC) at $38^{\circ} \mathrm{C}$ in tetrahydrofuran as an eluent by means of Tosoh 8000 series GPC system, and calibrated with standard polystyrenes.

The ${ }^{13} \mathrm{C}$ NMR spectra were recorded on a JEOL GX-400 spectrometer using deuterochloroform as a solvent and an internal standard $(\delta 77.03)$ at an ambient temperature $\left(c a .27^{\circ} \mathrm{C}\right)$. The operating conditions were same as those in the previous paper. ${ }^{7}$ The spectra were simulated to determine the $P_{m}$ according to the method previously reported. ${ }^{7}$ The typical examples of simulation parameters are as follows. Poly(DMF) derived from poly(MtBF) $\left(120^{\circ} \mathrm{C}\right) ; P_{m} 0.57$, for carbonyl carbon, chemical shifts 170.20 ( $\mathrm{mmmmmm}), 170.20$ ( $\mathrm{mmmmmr}$ ), 170.40 ( $\mathrm{mmmmrm}), 170.75$ (rmmmrm), 171.00 (mmmrmm), 171.25 ( $\mathrm{mmmrmr}), 171.50$ ( $\mathrm{mrm}$ rmm), and $171.60 \mathrm{ppm}$ (mrmrmr), half width $0.5 \mathrm{ppm}$; for methine carbon, chemical shifts, 46.00 ( $\mathrm{mmmmmm}), 46.45$ ( $\mathrm{mmmmmr}), 46.30$ (mmmmrm), 45.90 (rmmmrm), 44.50 (mmmr$\mathrm{mm}), 45.20$ ( $\mathrm{mmmrmr}), 45.00$ ( $\mathrm{mrmrmm})$, and $44.15 \mathrm{ppm}$ (mrmrmr), half width $1.0 \mathrm{ppm}$. Poly(DiPF) $\left(120^{\circ} \mathrm{C}\right) ; P_{m} 0.55$, for carbonyl carbon, chemical shifts 169.35 ( $\mathrm{mmmmmm}$ ), 169.35 ( $\mathrm{mmmmmr}), 169.85(\mathrm{mmmmrm}), 170.00$ (rmmmrm), $170.10(\mathrm{mmmrmm}), 170.25(\mathrm{mmm}-$ 
rmr), 170.45 (mrmrmm), and $170.65 \mathrm{ppm}$ ( $m r m r m r$ ), half width $0.52 \mathrm{ppm}$; for methine carbon, chemical shifts, $46.20(\mathrm{mmmm}), 46.20$ ( $\mathrm{mmmr}), 44.85(\mathrm{mmrm})$, and $44.70 \mathrm{ppm}(\mathrm{rmrm})$, half width $1.2 \mathrm{ppm}$.

\section{RESULTS AND DISCUSSION}

\section{Preparation of Poly (DRF)s}

To investigate the effect of polymerization temperature on $P_{m}$, poly(DRF)s were prepared at various temperatures. Table I summarizes the results of radical polymerization of DRFs in bulk or in solution in the presence of an appropriate initiator with or without UV irradiation in the temperature range of $0-160^{\circ} \mathrm{C}$. The effect of initiator, solvent, and monomer concentration on tacticity of the resulting polymers are neglected in the present study. All polymerizations gave high molecular weight polymers $\left(\bar{M}_{n}>10^{4}\right)$ enough to be used for determination of tacticity. DtBF which has the highest polymerization reactivity among the DRFs examined gave a polymer at $0^{\circ} \mathrm{C}$, whereas DMF necessitated high polymerization temperature to obtain a polymer because of its lower reactivity. These results agree with the previous result that DRFs with bulkier substituents have a higher polymerization reactivity. ${ }^{4}$

Polymerization Temperature Dependence of $P_{m}$ In the previous paper, ${ }^{7}$ it was reported that the spilitting due to the tacticity appeared in the signals for main-chain methine and carbonyl carbons in ${ }^{13} \mathrm{C}$ NMR spectra of poly(DRF)s, but the both spectra for poly(DtBF) and poly(MtBF) were too broad to predict their stereoregularity precisely. Therefore, these polymers were converted quantitatively into poly(DMF) by pyrolysis without any catalyst

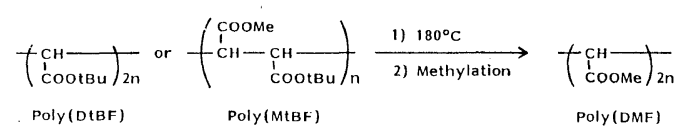

Scheme 1.

Table I. Results of radical polymerization of DRF

\begin{tabular}{|c|c|c|c|c|c|c|c|}
\hline$[\mathrm{DRF}]$ & [Initiator] & & Temp. & Time & Convn. & $\bar{M}_{n}$ & \\
\hline $\mathrm{moll}^{-1}$ & $\mathrm{moll}^{-1}$ & & ${ }^{\circ} \mathrm{C}$ & $\mathrm{h}$ & $\%$ & 人 & \\
\hline \multicolumn{8}{|l|}{ DtBF } \\
\hline 3.77 & DTBPO $(0.038)$ & Tol & 120 & 3 & 38.9 & 1.32 & 2.0 \\
\hline Bulk & $\mathrm{ACN} \quad(0.01)$ & None & 80 & 8 & 56.9 & 6.82 & 2.9 \\
\hline 2.0 & AIBN $\quad(0.02)$ & $\mathrm{Bz}$ & 40 & 30 & 22.1 & 11.6 & 2.3 \\
\hline 0.95 & DTBPO (0.02) & Tol & $0^{\mathrm{b}}$ & 50 & 1.7 & 1.78 & 1.7 \\
\hline \multicolumn{8}{|l|}{ MtBF } \\
\hline Bulk & DTBPO $(0.02)$ & None & 120 & 5 & 44.0 & 1.87 & 1.8 \\
\hline Bulk & $\mathrm{ACN} \quad(0.02)$ & None & 80 & 20 & 62.5 & 5.94 & 1.9 \\
\hline Bulk & AIBN $\quad(0.02)$ & None & 40 & 55 & 11.7 & 13.9 & 1.9 \\
\hline \multicolumn{8}{|l|}{ DiPF } \\
\hline Bulk & DTBPO (0.02) & None & 120 & 3 & 29.6 & 4.41 & 1.4 \\
\hline 3.46 & MAIB $\quad(0.02)$ & $\mathrm{Bz}$ & 60 & 1.5 & 15.7 & 13.8 & 1.6 \\
\hline 2.69 & DTBPO $(0.1)$ & Tol & $0^{\mathrm{b}}$ & 20 & 5.6 & 1.74 & 1.7 \\
\hline \multicolumn{8}{|l|}{ DMF } \\
\hline 5.78 & TBHPO (0.02) & Xyl & 160 & 10 & 14.0 & - & 一 \\
\hline 6.18 & DTBPO (0.062) & Tol & 120 & 12 & 25.5 & $(0.05)^{\mathrm{c}}$ & - \\
\hline 2.78 & $\mathrm{ACN} \quad(0.02)$ & $\mathrm{Bz}$ & 80 & 10 & 8.9 & - & - \\
\hline
\end{tabular}

a Tol, toluene; $\mathrm{Bz}$, benzene; $\mathrm{Xyl}, p$-xylene.

b Polymerized under UV irradiation.

${ }^{c}[\eta]$ in benzene at $30^{\circ} \mathrm{C}$ (in $\mathrm{dl} \mathrm{g}^{-1}$ ). 
and subsequent methylation (Scheme 1$),{ }^{7}$ prior to determination of the tacticity.

Figures 3-6 show the expanded ${ }^{13} \mathrm{C}$ NMR spectra for the methine and the carbonyl carbons of poly(DRF)s prepared under the conditions shown in Table I. It should be noted that the spectra in Figures 3 and 4 were observed as poly(DMF)s derived from poly(DtBF) and poly(MtBF), respectively, but not as poly(DtBF) and poly(MtBF) themselves. Poly(DiPF) was measured directly without any derivation, because quantitative transformation to poly(DMF) was very difficult. ${ }^{8}$

Previous studies ${ }^{6,7}$ with respect to triad sequences for poly(DRF) indicated that an $\mathrm{mm}$ triad signal appeared at a higher magnetic field than an $m r$ triad signal for the carbonyl carbon, and at a lower field for the methine carbon. The spectra of poly(DMF) derived from poly(DtBF) (Figure 3) clearly indicates that intensities of the $\mathrm{mm}$ triad regions of the both
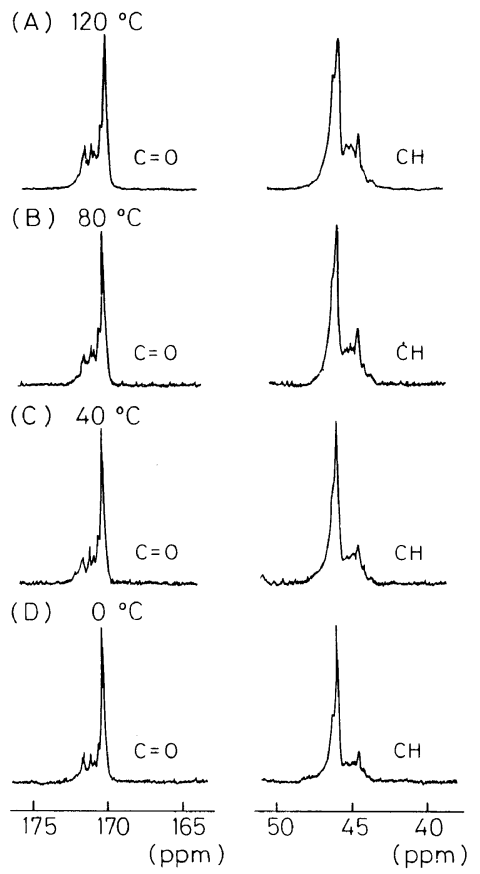

Figure 3. ${ }^{13} \mathrm{C}$ NMR spectra of main-chain methine and carbonyl carbons for poly(DMF) derived from poly(DtBF). Polymerization temperature: (A) $120^{\circ} \mathrm{C}$; (B) $80^{\circ} \mathrm{C}$; (C) $40^{\circ} \mathrm{C}$; (D) $0^{\circ} \mathrm{C}$. carbons, i.e., $169.5-170.6 \mathrm{ppm}$ and $45.6-48.0$ ppm for the carbonyl and methine carbons, respectively, increase with decreasing polymerization temperature. On the other hand, the peak intensities for the corresponding carbons of poly(DMF) derived from poly(MtBF) do

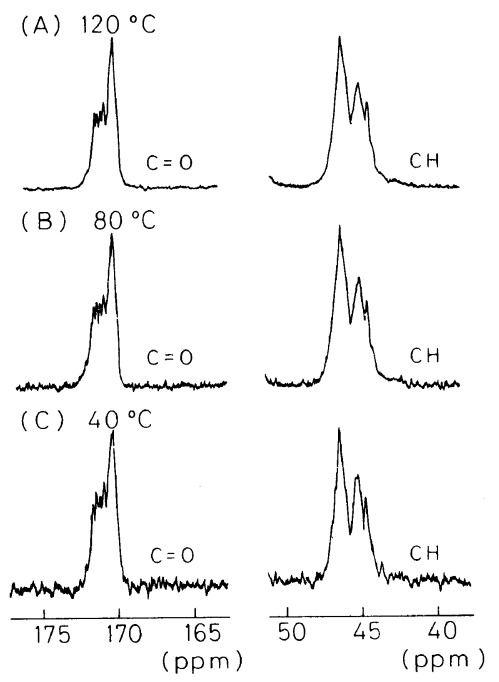

Figure 4. ${ }^{13} \mathrm{C}$ NMR spectra of main-chain methine and carbonyl carbons for poly(DMF) derived from poly(MtBF). Polymerization temperature: (A) $120^{\circ} \mathrm{C}$; (B) $80^{\circ} \mathrm{C}$; (C) $40^{\circ} \mathrm{C}$

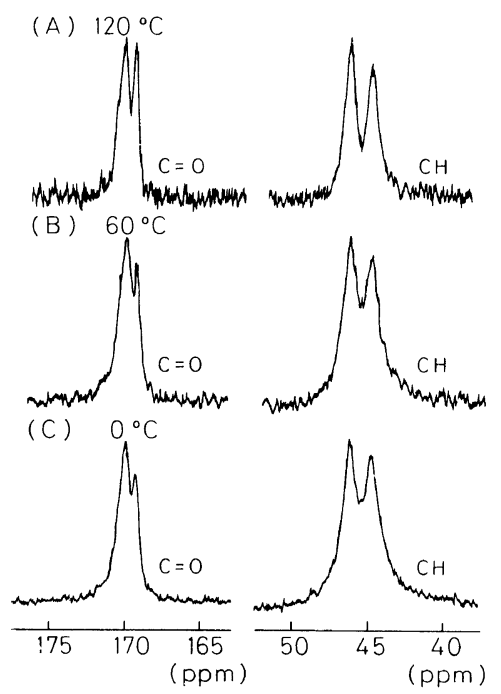

Figure 5. ${ }^{13} \mathrm{C}$ NMR spectra of main-chain methine and carbonyl carbons for poly(DiPF). Polymerization temperature: (A) $120^{\circ} \mathrm{C}$; (B) $60^{\circ} \mathrm{C}$; (C) $0^{\circ} \mathrm{C}$. 


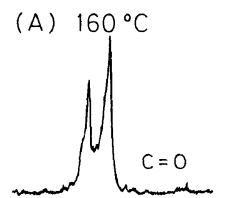

(B) $120^{\circ} \mathrm{C}$

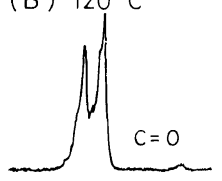

(C) $80^{\circ} \mathrm{C}$
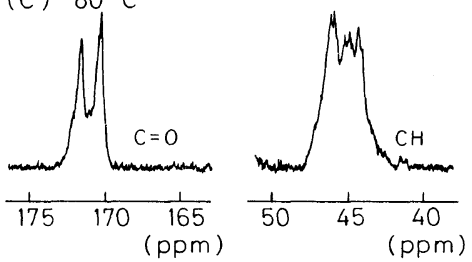

Figure 6. ${ }^{13} \mathrm{C}$ NMR spectra of main-chain methine and carbonyl carbons for poly(DMF). Polymerization temperature: (A) $160^{\circ} \mathrm{C}$; (B) $120^{\circ} \mathrm{C}$; (C) $80^{\circ} \mathrm{C}$.

not observed to change with the temperature as shown in Figure 4. In the cases of poly(DiPF) (Figure 5) and poly(DMF) (Figure 6), the $\mathrm{mm}$ triad regions slightly decrease with decreasing polymerization temperature.

In order to determine $P_{m}$ (probability of meso addition), the spectra of the methine and the carbonyl carbons of poly(DRF)s were simulated. The simulation was carried out with respect to heptad sequences except for the spectra for the methine carbon of poly(DiPF), which was simulated with pertad sequences because of their broad signals. ${ }^{6}$

Table II shows the $P_{m}$ determined by the simulation of the spectra in Figures 3-6. The $P_{m}$ values obtained were in the following order; DtBF $>$ MtBF $>$ DiPF $>$ DMF, agreeing with the results in the previous report. ${ }^{7}$ The $P_{m}$ for poly(DtBF) increased with the decrease in the polymerization temperature, i.e., it reached 0.80 at $0^{\circ} \mathrm{C}$, where an average fraction of the meso diad for the polymer was as high as 0.90 (Table II). On the other hand, the $P_{m}$ values for DiPF and DMF showed the opposite temperature dependence to DtBF, i.e., the $P_{m}$ decreases with decreasing temperature. No
Table II. $P_{m}$ values for polymerization of DRF and the probability of meso diad $([m])$ in poly(DRF)

\begin{tabular}{cccc}
\hline & $\begin{array}{c}\text { Polymerization } \\
\text { Demperature }\end{array}$ & & \\
& $P_{\boldsymbol{m}}$ & {$[\mathrm{m}]$} \\
\cline { 2 - 2 } DtBF & ${ }^{\circ} \mathrm{C}$ & & \\
& 120 & 0.68 & 0.84 \\
& 80 & 0.70 & 0.85 \\
MtBF & 40 & 0.75 & 0.88 \\
& 0 & 0.80 & 0.90 \\
DiPF & 120 & 0.57 & 0.79 \\
& 80 & 0.57 & 0.79 \\
& 40 & 0.57 & 0.79 \\
DMF & 120 & 0.55 & 0.78 \\
& 60 & 0.54 & 0.77 \\
& 0 & 0.53 & 0.77 \\
& 160 & 0.52 & 0.76 \\
& 120 & 0.50 & 0.75 \\
& 80 & 0.48 & 0.74 \\
\hline
\end{tabular}

dependence of the $P_{m}$ value was observed for polymerization of $\mathrm{MtBF}$.

\section{Activation Enthalpies and Entropies for meso and racemo Additions}

The differences in activation enthalpies and entropies for meso and racemo additions $\left(\Delta H_{m}^{\neq}-\Delta H_{r}^{\neq}\right.$and $\Delta S_{m}^{\neq}-\Delta S_{r}^{\neq}$, respectively) can be determined from the Arrhenius plot of the $P_{m}$ (eq 1 ), because $P_{m}$ is generally determined by the differences in free energies of activation for meso and racemo additions $\left(\Delta G_{m}^{\neq}-\Delta G_{r}^{\neq}\right)^{9,10}$

$$
\begin{aligned}
& \ln \left[P_{m} /\left(1-P_{m}\right)\right] \\
& =\ln \left(k_{m} / k_{r}\right) \\
& =-\left(\Delta G_{m}^{\neq}-\Delta G_{r}^{\neq}\right) / R T \\
& =-\left(\Delta H_{m}^{\neq}-\Delta H_{r}^{\ddagger}\right) / R T+\left(\Delta S_{m}^{\neq}-\Delta S_{r}^{\neq}\right) / R
\end{aligned}
$$

Where, $k_{m}$ and $k_{r}$ represent the rate constants for meso and racemo additions, respectively, and $R$ is the gas constant, and $T$ is the absolute polymerization temperature.

The plots of $\ln \left[P_{m} /\left(1-P_{m}\right)\right]$ against $1 / T$ gave a linear relation for each DRF (Figure 7). The 


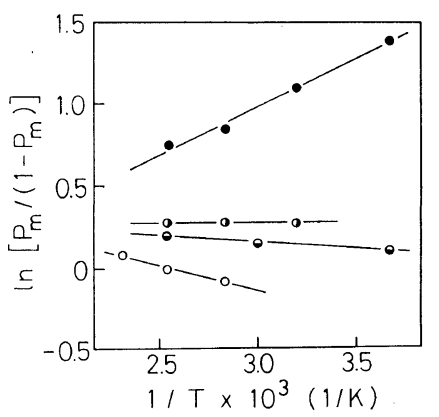

Figure 7. Arrhenius plots for the ratio of probabilities of meso addition to racemo addition for radical polymerization of $\operatorname{DtBF}(\bullet), \operatorname{MtBF}(\bullet), \operatorname{DiPF}(\bullet)$, and DMF (O).

Table III. Differences in activation enthalpies and entropies for meso and racemo additions in radical polymerization of DRF

\begin{tabular}{cccc}
\hline DRF & $\frac{\Delta H_{m}^{\neq}-\Delta H_{r}^{\neq}}{\mathrm{kJ} \mathrm{mol}^{-1}}$ & $\frac{\Delta S_{m}^{\neq}-\Delta S_{r}^{\neq}}{\mathrm{J} \mathrm{mol}^{-1} \mathrm{~K}^{-1}}$ & $\frac{\Delta G_{m}^{\neq}-\Delta G_{r}^{\neq}\left(60^{\circ} \mathrm{C}\right)}{\mathrm{kJ} \mathrm{mol}^{-1}}$ \\
\hline DtBF & -4.9 & -6.4 & -2.8 \\
MtBF & 0 & 2.3 & -0.77 \\
DiPF & 0.6 & 3.2 & -0.47 \\
DMF & 2.6 & 6.5 & 0.43 \\
\hline
\end{tabular}

$\Delta H_{m}^{\ddagger}-\Delta H_{r}^{\ddagger}$ and $\Delta S_{m}^{\neq}-\Delta S_{r}^{\neq}$determined are listed in Table III. The $\Delta H_{m}^{\neq}-\Delta H_{r}^{\neq}$value indicates that meso addition is preferred by enthalpy for polymerization of DtBF. On the other hand, the $\Delta H_{m}^{\neq}-\Delta H_{r}^{\ddagger}$ value is positive for polymerization of DMF. For polymerization of DiPF and MtBF, the $\Delta H_{m}^{\neq}-\Delta H_{r}^{\neq}$ values are the intermediate between those of DtBF and DMF. These results indicate that the $\Delta H_{m}^{\neq}-\Delta H_{r}^{\neq}$value depends remarkably on the structure of the ester substituents. The $\Delta S_{m}^{\neq}-\Delta S_{r}^{\neq}$velue is also dependent on the ester groups, i.e., it decreases along with the bulkiness of the ester substituents, indicating that the racemo addition is advantageous by entropy on account of the steric hindrance.

The $\Delta G_{m}^{\neq}-\Delta G_{r}^{\neq}$at $60^{\circ} \mathrm{C}$ were calculated from the $\Delta H_{m}^{\neq}-\Delta H_{r}^{\neq}$and the $\Delta S_{m}^{\neq}-\Delta S_{r}^{\neq}$ values, and are included in Table III. The values indicate that $\Delta G_{r}^{\neq}$is larger than $\Delta G_{m}^{\neq}$for polymerization of DtBF, while $\Delta G_{m}^{\neq}$value is larger for polymerization of DMF. It is surprising characteristics because the dependence on the structure of ester groups was scarcely observed for polymerization of alkyl methacrylates ${ }^{11}$ except for triphenylmethyl methacrylate, ${ }^{12}$ which shows the different behaviors originated from the formation of stable helical conformation in the polymerization solution. ${ }^{13}$ In the polymerization of DRFs, the effect of the structure of the ester substituents on $\Delta G_{m}^{\neq}-\Delta G_{r}^{\neq}$might be due to the steric factors peculiar to an attack of the 1,2-disubstituted ethylene toward the poly(substituted methylene) radical.

Furthermore, since the $P_{m} / P_{r}$ values are correlated to the $k_{m} / k_{r}$ values by using eq 1 , it is possible to determine the absolute values of $k_{m}$ and $k_{r}$ when the propagation rate constant is obtained. The detailed results are described in the next paper.

\section{REFERENCES}

1. T. Otsu, O. Ito, N. Toyoda, and S. Mori, Makromol. Chem., Rapid Commun., 2, 725 (1981).

2. T. Otsu, T. Yasuhara, K. Shiraishi, and S. Mori, Polym. Bull., 12, 449 (1984).

3. T. Otsu, T. Yasuhara, and A. Matsumoto, $J$. Macromol. Sci.-Chem., A25, 537 (1988).

4. A. Matsumoto, T. Tarui, and T. Otsu, Macromolecules, 23, 5102 (1990).

5. T. Otsu, B. Yamada, and T. Ishikawa, Macromolecules, 24, 415 (1991).

6. X. Wang, T. Komoto, I. Ando, and T. Otsu, Makromol. Chem., 189, 1845 (1988).

7. M. Yoshioka, A. Matsumoto, T. Otsu, and I. Ando, Polymer, in press.

8. T. Otsu, M. Yoshioka, A. Matsumoto, and K. Shiraishi, Polym. Bull., 26, 159 (1991).

9. F. A. Bovey, "Chain Structure and Conformation of Macromolecules," Academic Press, New York, N.Y., 1977.

10. P. Pino and U. W. Suter, Polymer, 17, 977 (1976).

11. K. Matsuzaki and T. Uryu, Kogyo Kagaku Zasshi, 70, 1864 (1967).

12. H. Yuki, K. Hatada, T. Niinomi, and Y. Kikuchi, $J$. Polym. Sci., B6, 753 (1968); Polym. J., 1, 36 (1970).

13. Y. Okamoto, K. Suzuki, K. Hatada, and H. Yuki, J. Am. Chem. Soc., 101, 6356 (1979); Y. Okamoto, H. Mohri, T. Nakano, and K. Hatada, ibid., 111, 5952 (1989). 